\title{
Construction of optical solitons of magneto-optic waveguides with anti-cubic law nonlinearity
}

\author{
Muhammad Imran Asjad ${ }^{1}$ - Naeem Ullah ${ }^{1} \cdot$ Hamood Ur Rehman² · Mustafa Inc 3,4,5 (D) \\ Received: 31 July 2021 / Accepted: 3 October 2021 / Published online: 24 October 2021 \\ (c) The Author(s), under exclusive licence to Springer Science+Business Media, LLC, part of Springer Nature 2021
}

\begin{abstract}
Two efficient integration schemes, new extended hyperbolic function and generalized tanh are employed to discover optical soliton solutions to magneto-optic waveguides that retains anti-cubic form of nonlinear refractive index. Bright, dark, periodic singular, singular, and combo soliton solutions have created. These solutions expose the comprehensive variety of soliton solutions.
\end{abstract}

Keywords Magneto-optic waveguides with anti-cubic law nonlinearity $\cdot$ New extended hyperbolic function method $\cdot$ Tanh method $\cdot$ Optical soliton solutions

\section{Introduction}

Optical solitons have a variety of applications in different optoelectronic tools such as optical couplers, birefringent fibers, polarization-preserving fibers, magneto-optic waveguides (MOW), metasurfaces and metamaterials as well as in wavelength division multiplexed structures and photonic crystal fibers. An abundant of results have been stated in all of these areas (Khater et al. 2020; Akinyemi 2021; Ali Akbar et al. 2021; Akinyemi et al. 2020; Akinyemi 2019; Raza 2020; Dotsch et al. 2005; Fedele et al. 2003; Guzman et al. 2014; Haider 2017; Hasegawa and Miyazaki 1992; Raza and Arshed 2020; Rehman et al. 2021a; b; c; Khan 2020; Kudryashov 2019; Qiu et al. 2019; Shoji and Mizumoto 2018; Wazwaz 2008; Yan et al. 2009; Zayed et al. 2020; Imran et al. 2020; Rehman et al. 2019; Zayed et al. 0000; Dai et al. 2017; Dai et al. 2020; Wang et al. 2018; Dai et al. 2019; Younas and Ren 2021; Bilal et al. 2021a; Bilal et al. 2021b; Bilal et al. 2021c; Bilal et al. 2021d). This paper describes dynamics behavior of solitons for MOW that retains anti-cubic (AC)

Mustafa Inc

minc@ firat.edu.tr

1 Department of Mathematics, University of Management and Technology, Lahore 54770, Pakistan

2 Department of Mathematics, University of Okara, Okara, Pakistan

3 Department of Computer Engineering, Biruni University, Istanbul, Turkey

4 Department of Mathematics, Firat University, Elazig, Turkey

5 Department of Medical Research, China Medical University Hospital, China Medical University, Taichung, Taiwan 
form of nonlinear refractive index (NLRI). The RI law was first suggested during 2003 and far ahead it was considered all across (Fedele et al. 2003). In this paper the construction of optical solitons solutions is made by using new extended hyperbolic function method (EHFM) and generalized tanh methods. In this work, purpose is to construct dark, singular and bright soliton solutions along with trigonometric and hyperbolic functions solutions for MOW with AC form of nonlinear RI. It is important that these discovered results are novel, correct and are being stated earliest in this paper. Likewise, it should be noted that the solitons discovered to MOW with AC law nonlinearity will be highly favorable in the fiber-optic communication technology.

This paper is structured as: Sect. 2 contains the governing medel. New extended hyperbolic function method and its application for MOW with AC nonlinearity are described in Sect. 3. Section 4 consists generalized tanh method and its application for the MOW with AC nonlinearity. Section 5 contains results and discussion and Sect. 6 occupys the conclusion of this work.

\section{Governing model}

A set of two nonlinear Schrödinger equations (NLSEs) in MOW with AC law nonlinearity is stated as

$$
\begin{aligned}
\iota q_{t} & +a_{1} q_{x x}+\left(\frac{b_{11}}{|q|^{4}}+b_{12}|q|^{2}+b_{13}|q|^{4}+\frac{c_{11}}{|r|^{4}}+c_{12}|r|^{2}+c_{13}|r|^{4}\right) q=Q_{1} r+\imath\left[\alpha_{1} q_{x}\right. \\
& \left.+\lambda_{1}\left(|q|^{2} q\right)_{x}+\theta_{1}|q|^{2} q_{x}+v_{1}\left(|q|^{2}\right)_{x} q\right], \\
u r_{t} & +a_{2} r_{x x}+\left(\frac{b_{21}}{|r|^{4}}+b_{22}|r|^{2}+b_{23}|r|^{4}+\frac{c_{21}}{|q|^{4}}+c_{22}|q|^{2}+c_{23}|q|^{4}\right) r=Q_{2} q+\imath\left[\alpha_{2} r_{x}\right. \\
& \left.+\lambda_{2}\left(|r|^{2} r\right)_{x}+\theta_{2}|r|^{2} r_{x}+v_{2}\left(|q|^{2}\right)_{x} r\right],
\end{aligned}
$$

where $a_{1}, b_{11}, b_{12}, b_{13}, c_{11}, c_{12}, c_{13}, Q_{1}, \alpha_{1}, \lambda_{1}, v_{1}$ and $\theta_{1}$ are constants, for $l=1,2$, while $l=\sqrt{-1}$. Where $q(x, t)$ and $r(x, t)$ are dependent variables of complex valued while $x$ and $t$ are spatial and temporal independent variables. Here $a_{1}$ is the coefficients of chromatic dispersion (CD), while coefficients of SPM are $b_{11}, b_{12}$ and $b_{13}$, whetre $c_{11}, c_{12}$ and $c_{13}$ are the terms of XPM. On the right side, $\alpha_{1}$ and $Q_{1}$ are the coefficients of inter-modal dispersions (IMD) and magneto-optic parameters while $\lambda_{1}$ gives the self-steepening (SS) terms. Lastly, $v_{1}$ and $\theta_{1}$ are the coefficients of nonlinear dispersion.

\subsection{Mathematical analysis}

From (1) and (2), we choose the supposition as follows

$$
\begin{aligned}
& q(x, t)=P_{1}(\eta) e^{l Y}, \\
& r(x, t)=P_{2}(\eta) e^{\imath Y},
\end{aligned}
$$

and 


$$
\eta=x-v t, \quad Y=-k x+w t+\theta_{0} .
$$

where $k, v, \theta_{0}$ and $w$ are the frequency, speed, phase constant, and wave number, respectively. Replacing (3) and (4) as well as (5) into (1) and (2). Real parts obtain

$$
\begin{aligned}
& a_{1} P_{1}^{\prime \prime}+\frac{b_{11}}{P_{1}^{3}}+b_{13} P_{1}^{5}+\frac{c_{11}}{P_{2}^{4}} P_{1}+c_{12} P_{1} P_{2}^{2}+\left[b_{12}-k\left(\lambda_{1}+\theta_{1}\right)\right] P_{1}^{3} \\
& \quad+c_{13} P_{1} P_{2}^{4}-\left[w+\alpha_{1} k+a_{1} k^{2}\right] P_{1}-Q_{1} P_{2}=0, \\
& a_{2} P_{2}^{\prime \prime}+\frac{b_{21}}{P_{2}^{3}}+b_{23} P_{2}^{5}+\frac{c_{21}}{P_{1}^{4}} P_{2}+c_{22} P_{2} P_{1}^{2}+\left[b_{22}-k\left(\lambda_{2}+\theta_{2}\right)\right] P_{2}^{3} \\
& \quad+c_{23} P_{2} P_{1}^{4}-\left[w+\alpha_{2} k+a_{2} k^{2}\right] P_{2}-Q_{2} P_{1}=0,
\end{aligned}
$$

imaginary parts give

$$
\begin{aligned}
& \left(v+2 a_{1} k+\alpha_{1}\right) P_{1}^{\prime}+\left(3 \lambda_{1}+2 v_{1}+\theta_{1}\right) P_{1}^{2} P_{1}^{\prime}=0, \\
& \left(v+2 a_{2} k+\alpha_{2}\right) P_{2}^{\prime}+\left(3 \lambda_{2}+2 v_{2}+\theta_{2}\right) P_{2}^{2} P_{2}^{\prime}=0 .
\end{aligned}
$$

Using linearly independent principle, we obtain from (8) and (9).

$$
\begin{gathered}
v=-\left(2 a_{1} k+\alpha_{1}\right), \\
3 \lambda_{1}+2 v_{1}+\theta_{1}=0,
\end{gathered}
$$

and

$$
\begin{gathered}
v=-\left(2 a_{2} k+\alpha_{2}\right), \\
3 \lambda_{2}+2 v_{2}+\theta_{2}=0,
\end{gathered}
$$

we get frequency of soliton using (10) and (12),

$$
k=\frac{\left(\alpha_{2}-\alpha_{1}\right)}{2\left(a_{1}-a_{2}\right)},
$$

provided

$$
a_{1} \neq a_{2}, \quad \alpha_{2} \neq \alpha_{1} \text {. }
$$

Set

$$
P_{2}(\eta)=\chi P_{1}(\eta)
$$

here $\chi$ is a constant and $\chi \neq 1$. Next, (6) and (7) convert to

$$
\begin{aligned}
& a_{1} P_{1}^{3} P_{1}^{\prime \prime}+\left(b_{11}+\frac{c_{11}}{\chi^{4}}\right)-\left(w+\alpha_{1} k+a_{1} k^{2}+Q_{1} \chi\right) P_{1}^{4} \\
& \quad+\left[c_{12} \chi^{2}+b_{12}-k\left(\lambda_{1}+\theta_{1}\right)\right] P_{1}^{6}+\left(b_{13}+c_{13} \chi^{4}\right) P_{1}^{8}=0,
\end{aligned}
$$




$$
\begin{aligned}
& a_{2} P_{1}^{3} P_{1}^{\prime \prime}+\left(\frac{b_{21}}{\chi^{3}}+c_{21} \chi\right)-\left[\left(w+\alpha_{2} k+a_{2} k^{2}\right) \chi+Q_{2}\right] P_{1}^{4} \\
& \quad+c_{22} \chi^{2}+\left[b_{22}-k\left(\lambda_{2}+\theta_{2}\right) \chi^{3}\right] P_{1}^{6}+\left(b_{23} \chi^{5}+c_{23} \chi\right) P_{1}^{8}=0 .
\end{aligned}
$$

Using the constraint conditions

$$
\begin{gathered}
a_{2}=a_{1} \chi, \\
b_{11}+\frac{c_{11}}{\chi^{4}}+\frac{b_{21}}{\chi^{3}}+c_{21} \chi, \\
w+\alpha_{1} k+a_{1} k^{2}+Q_{1} \chi=\left[w+\alpha_{2} k+a_{2} k^{2}\right] \chi+Q_{2}, \\
c_{12} \chi^{2}+b_{12}-k\left(\lambda_{1}+\theta_{1}\right)=c_{22} \chi+\left[b_{22}-k\left(\lambda_{2}+\theta_{2}\right)\right] \chi^{3}, \\
b_{13}+c_{13} \chi^{4}=b_{23} \chi^{5}+c_{23} \chi,
\end{gathered}
$$

(17) and (18) are similar to see. Next, the wave number $w$ is attained from (19) and (21),

$$
w=\frac{\chi Q_{1}-Q_{2}-k\left(\chi \alpha_{2}-\alpha_{1}\right)}{\chi_{1}}
$$

Balancing $P_{1}^{3} P_{1}^{\prime \prime}$ and $P_{1}^{8}$ in Eq. (17), yields $N=\frac{1}{2}$,

$$
P_{1}(\eta)=[U(\eta)]^{\frac{1}{2}} .
$$

(17) converts to

$$
4\left(\Lambda_{0}+\Lambda_{2} U^{2}+\Lambda_{3} U^{3}+\Lambda_{4} U^{4}\right)+a_{1}\left(2 U U^{\prime \prime}-U^{\prime 2}\right)=0
$$

where

$$
\begin{aligned}
& \Lambda_{0}=b_{11}+\frac{c_{11}}{\chi^{4}}, \\
& \Lambda_{2}=-\left(w+\alpha_{1} k+a_{1} k^{2}+Q_{1} \chi\right), \\
& \Lambda_{3}=c_{12} \chi^{2}+b_{12}-k\left(\lambda_{1}+\theta_{1}\right), \\
& \Lambda_{4}=b_{13}+c_{13} \chi^{4} .
\end{aligned}
$$

\section{New EHFM}

The new EHFM (Shang 2008; Shang et al. 2008; Nestor et al. 2020) have some phases as follows

Form 1: Let PDE as given in (1)-(2) with the wave transformation in (4)-(5) along with (6) using wave transformation ODE is obtained as in (26). We assume that (26) has a solution in the next form 


$$
U(\eta)=\sum_{i=0}^{N} F_{i} \Phi^{i}(\eta)
$$

where $F_{i}(i=(1,2,3, \ldots . . N))$ are constants and $\Phi(\eta)$ admits the ODE in next form, as

$$
\frac{d \Phi}{d \eta}=\Phi \sqrt{\tau+\Theta \Phi^{2}}, \tau, \Theta \in R
$$

By using balancing rule in (26) the value of $N$ is found. Replacing (28) into (26) with (29), offers a set of equations for $F_{i}(i=(0,1,2,3, \ldots . N))$. On solving this set, we yield set of solutions that admits (29), so

Set 1: When $\tau>0$ and $\Theta>0$,

$$
\Phi(\eta)=-\sqrt{\frac{\tau}{\Theta}} \operatorname{csch}\left(\sqrt{\tau}\left(\eta+\eta_{0}\right)\right) .
$$

Set 2: When $\tau<0$ and $\Theta>0$,

$$
\Phi(\eta)=\sqrt{\frac{-\tau}{\Theta}} \sec \left(\sqrt{-\tau}\left(\eta+\eta_{0}\right)\right) .
$$

Set 3: When $\tau>0$ and $\Theta<0$,

$$
\Phi(\eta)=\sqrt{\frac{\tau}{-\Theta}} \operatorname{sech}\left(\sqrt{\tau}\left(\eta+\eta_{0}\right)\right) .
$$

Set 4: When $\tau<0$ and $\Theta>0$,

$$
\Phi(\eta)=\sqrt{\frac{-\tau}{\Theta}} \csc \left(\sqrt{-\tau}\left(\eta+\eta_{0}\right)\right) .
$$

Set 5: When $\tau>0$ and $\Theta=0$,

$$
\Phi(\eta)=\exp \left(\sqrt{\tau}\left(\eta+\eta_{0}\right)\right) .
$$

Set 6: When $\tau<0$ and $\Theta=0$,

$$
\Phi(\eta)=\cos \left(\sqrt{-\tau}\left(\eta+\eta_{0}\right)\right)+\imath \sin \left(\sqrt{-\tau}\left(\eta+\eta_{0}\right)\right) .
$$

Set 7: When $\tau=0$ and $\Theta>0$,

$$
\Phi(\eta)= \pm \frac{1}{\left(\sqrt{\Theta}\left(\eta+\eta_{0}\right)\right)} .
$$

Set 8: When $\tau=0$ and $\Theta<0$,

$$
\Phi(\eta)= \pm \frac{l}{\left(\sqrt{-\Theta}\left(\eta+\eta_{0}\right)\right)} .
$$

Form 2: Using the same pattern as previous, we adopt that $\Phi(\eta)$ admits the ODE as follows 


$$
\frac{d \Phi}{d \eta}=\tau+\Theta \Phi^{2}, \tau, \Theta \in R
$$

Substituting (28) into (26) along with (38) along value of $N$, makes a set of equations as well the values of $F_{i}(i=1,2,3, \ldots N)$.

Let the (38) accepts the solutions, so

Set 1: When $\tau \Theta>0$,

$$
\Phi(\eta)=\operatorname{sgn}(\tau) \sqrt{\frac{\tau}{\Theta}} \tan \left(\sqrt{\tau \Theta}\left(\eta+\eta_{0}\right)\right) .
$$

Set 2: When $\tau \Theta>0$,

$$
\Phi(\eta)=-\operatorname{sgn}(\tau) \sqrt{\frac{\tau}{\Theta}} \cot \left(\sqrt{\tau \Theta}\left(\eta+\eta_{0}\right)\right) .
$$

Set 3: When $\tau \Theta<0$,

$$
\Phi(\eta)=\operatorname{sgn}(\tau) \sqrt{\frac{\tau}{-\Theta}} \tanh \left(\sqrt{-\tau \Theta}\left(\eta+\eta_{0}\right)\right) .
$$

Set 4: When $\tau \Theta<0$,

$$
\Phi(\eta)=\operatorname{sgn}(\tau) \sqrt{\frac{\tau}{-\Theta}} \operatorname{coth}\left(\sqrt{-\tau \Theta}\left(\eta+\eta_{0}\right)\right) .
$$

Set 5: When $\tau=0$ and $\Theta>0$,

$$
\Phi(\eta)=-\frac{1}{\Theta\left(\eta+\eta_{0}\right)} .
$$

Set 6: When $\tau \in R$ and $\Theta=0$,

$$
\Phi(\eta)=\tau\left(\eta+\eta_{0}\right) .
$$

Note: $s g n$ is the famous sign functions.

\subsection{Application of the new EHFM}

Form 1: Here, we employ the new EHFM for the MOW with AC form of NLRI to construct the optical solitons solutions. Using balancing method in (26), gives $N=1$, so (28) gives

$$
U(\eta)=F_{0}+F_{1} \Phi(\eta)
$$

where $F_{0}$ and $F_{2}$ are constants. Replacing (45) into (26) and equating the coefficients polynomials of $\Phi(\eta)$ to zero, we get a set of equations in $F_{0}, F_{1}, \tau, \Theta$ and $\Lambda_{0}$.

On resolving the set of equations, we attain 


$$
\begin{aligned}
F_{0} & =-\frac{3 \Lambda_{3}}{8 \Lambda_{4}}, \quad F_{1}=F_{1}, \\
\tau & =\frac{-32 \Lambda_{2}+\frac{9 \Lambda_{3}^{2}}{\Lambda_{4}}}{8 a_{1}}, \Theta=-\frac{4 F_{1}^{2} \Lambda_{4}}{3 a_{1}} . \\
\Lambda_{0} & =-\frac{9\left(15 \Lambda_{3}^{4}+64 \Lambda_{2} \Lambda_{3}^{2} \Lambda_{4}\right)}{4096 \Lambda_{4}^{3}} .
\end{aligned}
$$

Set 1: When $\tau>0$ and $\Theta>0$,

$$
\begin{gathered}
q_{1}(x, t)=\left(-\frac{3 \Lambda_{3}}{8 \Lambda_{4}}+F_{1}\left(-\sqrt{\frac{3\left(-9 \Lambda_{3}^{2}+32 \Lambda_{2} \Lambda_{4}\right)}{32 F_{1}^{2} \Lambda_{4}^{2}}}\right.\right. \\
\left.\left.\operatorname{csch}\left(\sqrt{\frac{-32 \Lambda_{2}+\frac{9 \Lambda_{3}^{2}}{\Lambda_{4}}}{8 a_{1}}}\left(\eta+\eta_{0}\right)\right)\right)\right)^{\frac{1}{2}} \times e^{l\left(-k w+w t+\theta_{0}\right)} . \\
r_{1}=\chi q_{1}(x, t) .
\end{gathered}
$$

Set 2: When $\tau<0$ and $\Theta>0$,

$$
\begin{gathered}
q_{2}(x, t)=\left(-\frac{3 \Lambda_{3}}{8 \Lambda_{4}}+F_{1}\left(\sqrt{-\frac{3\left(-9 \Lambda_{3}^{2}+32 \Lambda_{2} \Lambda_{4}\right)}{32 F_{1}^{2} \Lambda_{4}^{2}}}\right.\right. \\
\left.\left.\sec \left(\sqrt{-\frac{-32 \Lambda_{2}+\frac{9 \Lambda_{3}^{2}}{\Lambda_{4}}}{8 a_{1}}}\left(\eta+\eta_{0}\right)\right)\right)\right)^{\frac{1}{2}} \times e^{l\left(-k w+w t+\theta_{0}\right)} . \\
r_{2}=\chi q_{2}(x, t) .
\end{gathered}
$$

Set 3: When $\tau>0$ and $\Theta<0$,

$$
\begin{gathered}
q_{3}(x, t)=\left(-\frac{3 \Lambda_{3}}{8 \Lambda_{4}}+F_{1}\left(\sqrt{-\frac{3\left(-9 \Lambda_{3}^{2}+32 \Lambda_{2} \Lambda_{4}\right)}{32 F_{1}^{2} \Lambda_{4}^{2}}}\right.\right. \\
\operatorname{sech}\left(\sqrt{\left.\left.-\frac{-32 \Lambda_{2}+\frac{9 \Lambda_{3}^{2}}{\Lambda_{4}}}{8 a_{1}}\left(\eta+\eta_{0}\right)\right)\right)} \times e^{l\left(-k w+w t+\theta_{0}\right)} .\right. \\
r_{3}=\chi q_{3}(x, t) .
\end{gathered}
$$

Set 4: When $\tau<0$ and $\Theta<0$, 


$$
\begin{gathered}
q_{4}(x, t)=\left(-\frac{3 \Lambda_{3}}{8 \Lambda_{4}}+F_{1}\left(\sqrt{-\frac{3\left(-9 \Lambda_{3}^{2}+32 \Lambda_{2} \Lambda_{4}\right)}{32 F_{1}^{2} \Lambda_{4}^{2}}}\right.\right. \\
\csc \left(\sqrt{\left.\left.\left.-\frac{-32 \Lambda_{2}+\frac{9 \Lambda_{3}^{2}}{\Lambda_{4}}}{8 a_{1}}\left(\eta+\eta_{0}\right)\right)\right)\right)^{\frac{1}{2}} \times e^{\imath\left(-k w+w t+\theta_{0}\right)} .}\right. \\
r_{4}=\chi q_{4}(x, t) .
\end{gathered}
$$

Set 5: When $\tau=0$ and $\Theta>0$,

$$
\begin{gathered}
q_{5}(x, t)=\left(-\frac{3 \Lambda_{3}}{8 \Lambda_{4}}+F_{1}\left( \pm \frac{1}{\sqrt{-\frac{4 F_{1}^{2} \Lambda_{4}}{3 a_{1}}\left(\eta+\eta_{0}\right)}}\right)\right)^{\frac{1}{2}} \times e^{l\left(-k w+w t+\theta_{0}\right)} . \\
r_{5}=\chi q_{5}(x, t) .
\end{gathered}
$$

Set 6: When $\tau=0$ and $\Theta<0$,

$$
\begin{gathered}
q_{6}(x, t)=\left(-\frac{3 \Lambda_{3}}{8 \Lambda_{4}}+F_{1}\left( \pm \frac{l}{\sqrt{\frac{4 F_{1}^{2} \Lambda_{4}}{3 a_{1}}}\left(\eta+\eta_{0}\right)}\right)\right)^{\frac{1}{2}} \times e^{l\left(-k w+w t+\theta_{0}\right)}, \\
r_{6}=\chi q_{6}(x, t) .
\end{gathered}
$$

where $\eta=(x-v t)$, and $Y=-k x+w t+\theta_{0}$.

\section{Form 2:}

Operating balancing rule in (26), gives $N=1$, so (28) brings to

$$
U(\eta)=F_{0}+F_{1} \Phi(\eta),
$$

where $F_{0}$ and $F_{1}$ are constants. Replacing (59) into (26) and equating the coefficients of polynomials of $\Phi(\eta)$ to zero, we get a set of equations in $F_{0}, F_{1}, \tau, \Theta$, and $\Lambda_{0}$.

On resolving the set of equations, we attain

$$
\begin{gathered}
F_{0}=-\frac{3 \Lambda_{3}}{8 \Lambda_{4}}, \quad F_{1}=F_{1}, \quad a_{1}=-\frac{4 F_{1}^{2} \Lambda_{4}}{3 \Theta^{2}}, \\
\tau=\frac{3 \Theta\left(-9 \Lambda_{3}^{2}+32 \Lambda_{2} \Lambda_{4}\right)}{64 F_{1}^{2} \Lambda_{4}^{2}}, \Theta=\Theta,
\end{gathered}
$$




$$
\Lambda_{0}=-\frac{3\left(9 \Lambda_{3}^{2}-96 \Lambda_{2} \Lambda_{3}^{2} \Lambda_{4}+256 \Lambda_{2}^{2} \Lambda_{4}^{2}\right)}{1024 \Lambda_{4}^{3}}
$$

Set 1: When $\tau \Theta>0$,

$$
\begin{gathered}
q_{7}(x, t)=\left(-\frac{3 \Lambda_{3}}{8 \Lambda_{4}}+F_{1}\left(\Gamma \sqrt{\frac{3\left(-9 \Lambda_{3}^{2}+32 \Lambda_{2} \Lambda_{4}\right)}{64 F_{1}^{2} \Lambda_{4}^{2}}}\right.\right. \\
\left.\left.\tan \left(\sqrt{\frac{3 \Theta^{2}\left(-9 \Lambda_{3}^{2}+32 \Lambda_{2} \Lambda_{4}\right)}{64 F_{1}^{2} \Lambda_{4}^{2}}\left(\eta+\eta_{0}\right)}\right)\right)\right)^{\frac{1}{2}} \times e^{l\left(-k w+w t+\theta_{0}\right)} . \\
r_{7}=\chi q_{7}(x, t) .
\end{gathered}
$$

Set 2: When $\tau \Theta>0$,

$$
\begin{gathered}
q_{8}(x, t)=\left(-\frac{3 \Lambda_{3}}{8 \Lambda_{4}}+F_{1}\left(-\Gamma \sqrt{\frac{3\left(-9 \Lambda_{3}^{2}+32 \Lambda_{2} \Lambda_{4}\right)}{64 F_{1}^{2} \Lambda_{4}^{2}}}\right.\right. \\
\cot \sqrt{\left.\left.\left.\frac{3 \Theta^{2}\left(-9 \Lambda_{3}^{2}+32 \Lambda_{2} \Lambda_{4}\right)}{64 F_{1}^{2} \Lambda_{4}^{2}}\left(\eta+\eta_{0}\right)\right)\right)\right)^{\frac{1}{2}} \times e^{l\left(-k w+w t+\theta_{0}\right)} .} \\
r_{8}=\chi q_{8}(x, t) .
\end{gathered}
$$

Set 3: When $\tau \Theta<0$,

$$
\begin{gathered}
q_{9}(x, t)=\left(-\frac{3 \Lambda_{3}}{8 \Lambda_{4}}+F_{1}\left(\Gamma \sqrt{-\frac{3\left(-9 \Lambda_{3}^{2}+32 \Lambda_{2} \Lambda_{4}\right)}{64 F_{1}^{2} \Lambda_{4}^{2}}}\right.\right. \\
\left.\left.\tanh \left(\sqrt{-\frac{3 \Theta^{2}\left(-9 \Lambda_{3}^{2}+32 \Lambda_{2} \Lambda_{4}\right)}{64 F_{1}^{2} \Lambda_{4}^{2}}\left(\eta+\eta_{0}\right)}\right)\right)\right)^{\frac{1}{2}} \times e^{l\left(-k w+w t+\theta_{0}\right)} . \\
r_{9}=\chi q_{9}(x, t) .
\end{gathered}
$$

Set 4: When $\tau \Theta<0$,

$$
\begin{aligned}
q_{10}(x, t)= & \left(-\frac{3 \Lambda_{3}}{8 \Lambda_{4}}+F_{1}\left(\Gamma \sqrt{-\frac{3\left(-9 \Lambda_{3}^{2}+32 \Lambda_{2} \Lambda_{4}\right)}{64 F_{1}^{2} \Lambda_{4}^{2}}}\right.\right. \\
& \left.\left.\operatorname{coth}\left(\sqrt{-\frac{3 \Theta^{2}\left(-9 \Lambda_{3}^{2}+32 \Lambda_{2} \Lambda_{4}\right)}{64 F_{1}^{2} \Lambda_{4}^{2}}\left(\eta+\eta_{0}\right)}\right)\right)\right)^{\frac{1}{2}} \times e^{l\left(-k w+w t+\theta_{0}\right)} .
\end{aligned}
$$




$$
r_{10}=\chi q_{10}(x, t) \text {. }
$$

Set 5: When $\tau=0$ and $\Theta>0$,

$$
\begin{gathered}
q_{11}(x, t)=\left(-\frac{3 \Lambda_{3}}{8 \Lambda_{4}}+F_{1}\left(-\frac{1}{\Theta\left(\eta+\eta_{0}\right)}\right)\right)^{\frac{1}{2}} \times e^{l\left(-k w+w t+\theta_{0}\right)} . \\
r_{11}=\chi q_{11}(x, t) .
\end{gathered}
$$

\section{The generalized Tanh method Ullah et al. (2020)}

Let the PDE, as given in (1)-(2) with the wave transformation in (3)-(4) along with (5) using wave transformation ODE is obtained as in (26). We assume that (26) has a solution, as

$$
U(\eta)=\sum_{i=0}^{N} F_{i} \Phi^{i}(\eta),
$$

here, $F_{i}(i=(1,2,3, \ldots N))$ are constants and $\Phi(\eta)$

$$
\frac{d \Phi}{d \eta}=h+\Phi^{2}(\eta)
$$

By using balancing rule in (26) the value of $N$ is found. Replacing (72) into (26) with (73), offers a set of equations for $F_{i}(i=(0,1,2,3, \ldots N))$. On solving this set, we yield set of solutions that admits (73), as follows

Consider the solutions of (21) are, as

Case 1 . If $h<0$, then

$$
\Phi(\eta)=-\sqrt{-h} \tanh (\sqrt{-h} \eta)
$$

and

$$
\Phi(\eta)=-\sqrt{-h} \operatorname{coth}(\sqrt{-h} \eta)
$$

Case 2. If $h=0$, then

$$
\Phi(\eta)=-\frac{1}{\eta} .
$$

Case 3. If $h>0$, then

$$
\Phi(\eta)=\sqrt{h} \tan (\sqrt{h} \eta),
$$

and

$$
\Phi(\eta)=-\sqrt{h} \cot (\sqrt{h} \eta) .
$$




\subsection{Application of the generalized Tanh method}

According the balancing rule in (26), we get, $N=1,(72)$ reduces to

$$
U(\eta)=F_{0}+F_{1} \Phi(\eta)
$$

Putting (79) and (73) in (26) produces a polynomial in form of $\Phi(\eta)$. We get a system on making a comparison of the coefficients of $\Phi(\eta)$ to zero, after solving it, we get solutions as

$$
\begin{aligned}
& F_{0}=-\frac{3 \Lambda_{3}}{8 \Lambda_{4}}, \quad F_{1}=F_{1}, \quad a_{1}=-\frac{4}{3} F_{1}^{2} \Lambda_{4}, \\
& \Lambda_{2}=-\frac{9 \lambda_{3}^{2}}{32 \Lambda_{4}}+\frac{2}{3} h F_{1}^{2} \Lambda_{4}, \quad \Lambda_{0}=-\frac{\left(9 \Lambda_{3}^{2}+64 h F_{1}^{2} \Lambda_{4}^{2}\right)^{2}}{12288 \Lambda_{4}^{3}} .
\end{aligned}
$$

If $h<0$, then

$$
\begin{gathered}
q_{1}(x, t)=\left(\left(-\frac{3 \Lambda_{3}}{8 \Lambda_{4}}+F_{1} \sqrt{-h} \tanh (\sqrt{-h} \eta)\right)\right)^{\frac{1}{2}} \times e^{l\left(-k w+w t+\theta_{0}\right)}, \\
r_{1}=\chi q_{1}(x, t)
\end{gathered}
$$

or

$$
\begin{gathered}
q_{2}(x, t)=\left(\left(-\frac{3 \Lambda_{3}}{8 \Lambda_{4}}+F_{1} \sqrt{-h} \operatorname{coth}(\sqrt{-h} \eta)\right)\right)^{\frac{1}{2}} \times e^{l\left(-k w+w t+\theta_{0}\right)} . \\
r_{2}=\chi q_{2}(x, t) .
\end{gathered}
$$

If $h=0$, then

$$
\begin{gathered}
q_{3}(x, t)=\left(-\frac{3 \Lambda_{3}}{8 \Lambda_{4}}+F_{1}\left(-\frac{1}{\eta}\right)\right)^{\frac{1}{2}} \times e^{l\left(-k w+w t+\theta_{0}\right)} . \\
r_{3}=\chi q_{3}(x, t) .
\end{gathered}
$$

If $h>0$, then

$$
\begin{gathered}
q_{4}(x, t)=\left(\left(-\frac{3 \Lambda_{3}}{8 \Lambda_{4}}+F_{1} \sqrt{h} \tan (\sqrt{h} \eta)\right)\right)^{\frac{1}{2}} \times e^{l\left(-k w+w t+\theta_{0}\right)}, \\
r_{4}=\chi q_{4}(x, t) .
\end{gathered}
$$

or

$$
q_{5}(x, t)=\left(\left(-\frac{3 \Lambda_{3}}{8 \Lambda_{4}}+F_{1} \sqrt{h} \cot (\sqrt{h} \eta)\right)\right)^{\frac{1}{2}} \times e^{l\left(-k w+w t+\theta_{0}\right)}
$$


(a)

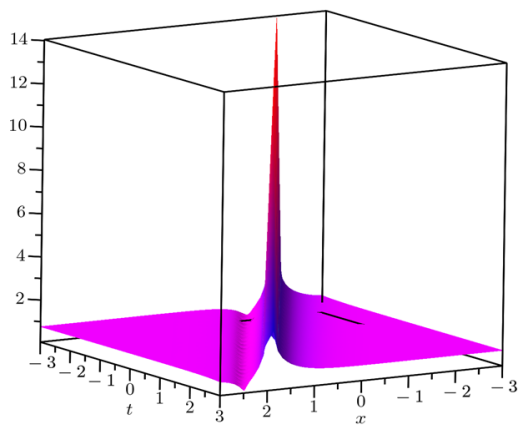

(a-1)

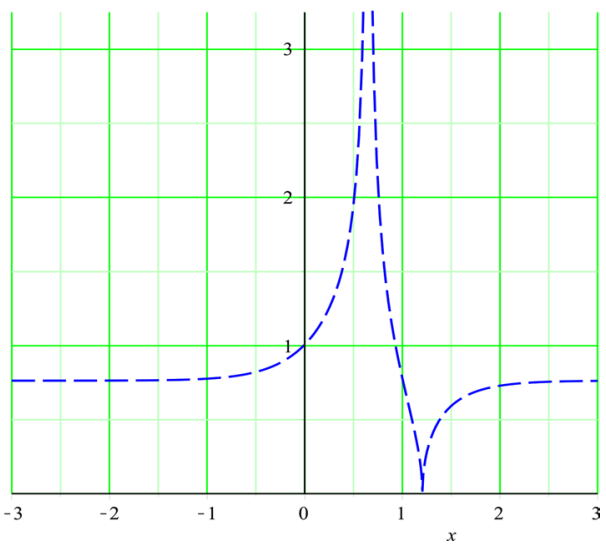

Fig. 1 a 3-d graph of (47) with $a_{1}=1.75, \Lambda_{2}=-1.3, \Lambda_{3}=1.4, \Lambda_{4}=-0.9, v=0.65, k=0.8, \theta_{1}=0.56$, $\Lambda_{1}=1.2, F_{1}=0.6, w=2$. (a-1) 2 -d plot of (47) with $t=1$

(b)

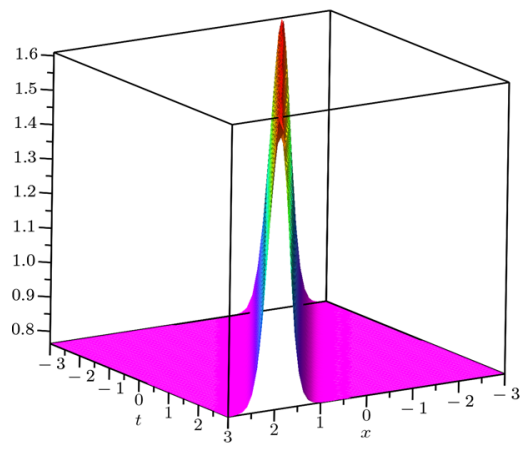

(b-1)

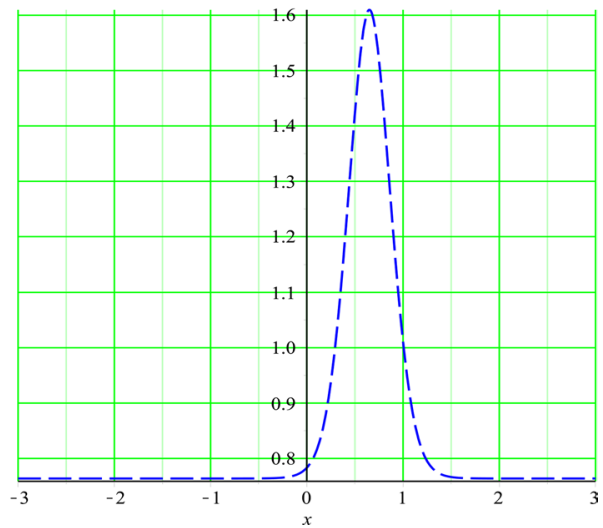

Fig. 2 b 3-d graph of (51) with $a_{1}=1.77, \Lambda_{2}=-1.2, \Lambda_{3}=1.6, \Lambda_{4}=-0.9, v=0.65, k=0.8, \theta_{1}=0.86$ , $\Lambda_{1}=1.2, F_{1}=0.6, w=2$. (b-1) 2 -d plot of (51) with $t=1$

$$
r_{5}=\chi q_{5}(x, t)
$$

where $\eta=(x-v t)$, and $Y=-k x+w t+\theta_{0}$.

\section{Results and discussions}

The results of this paper will be valuable for researchers to study the most noticeable applications of the MOW equation in optic fibers. Figures 1, 2, 3, 4, 5, 6 clearly reveals the surfaces of the solution acquired for 3-dim and 2-dim plots, with selection of suitable parameters for the MOW equation. Likewise, 3D plots provide us to model and 
(c)

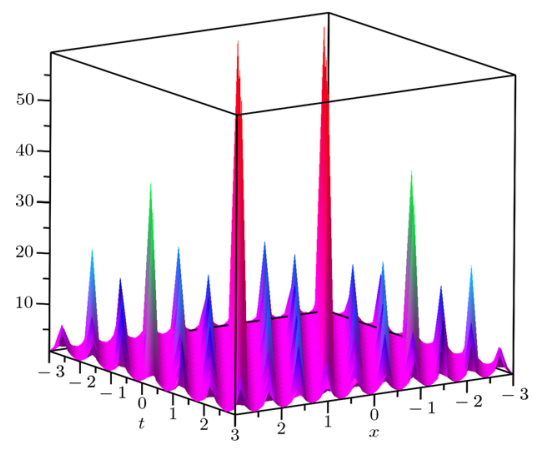

(c-1)

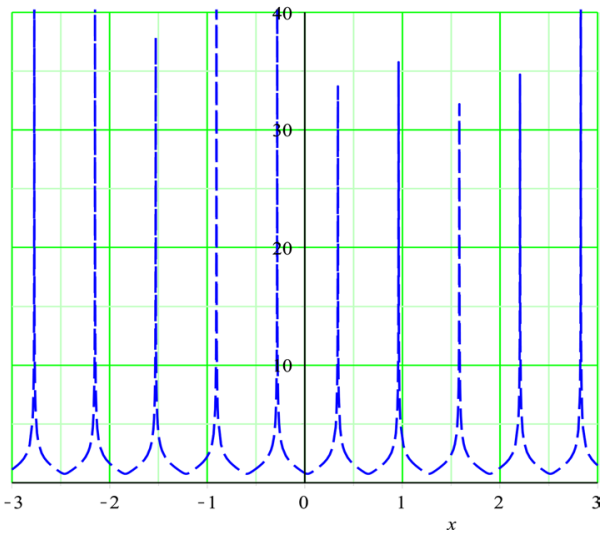

Fig. 3 c 3-d graph of (62) with $\Lambda_{2}=1.1, \Lambda_{3}=1.3, \Lambda_{4}=1.9, \Theta=1.75, v=0.65, k=0.9, \theta_{1}=0.56$ , $\Lambda_{1}=1.3, F_{1}=1.6, w=2, \Gamma=0.44$. (c-1) 2-d plot of (64) with $t=1$

(d)

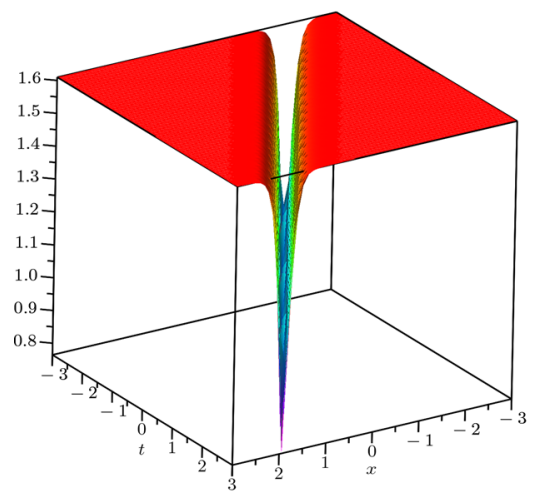

(d-1)

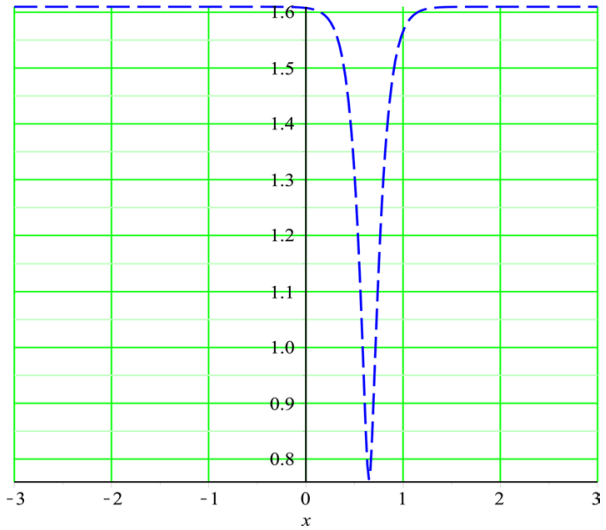

Fig. 4 d 3-d graph of (64) with $\Lambda_{2}=2.1, \Lambda_{3}=1.7, \Lambda_{4}=-1.9, \Theta=0.75, v=0.65, k=0.7, \theta_{1}=1.66$ , $\Lambda_{1}=1.1, F_{1}=0.6, w=2, \Gamma=0.35$. (d-1) 2-d plot of (64) with $t=1$

exhibit accurate physical behavior. Through this study, we consider the optical soliton solutions to the nonlinear MOW model using new EHFM and tanh method. The authors proposed different analytic approach in newly issued article and reported some fascinating findings. The authors can understand from all the graphs that the new EHFM and generalized tanh are very effectual and more specific in assessing the equation under consideration. Figures 1 and 2 designate the solutions stated by (47) and (51) which are bright and singular respectively. Figures 3 and 4 are the graphical representations of the solutions stated by (62) and (64) which are dark and singular solitons respectively. While Figures 5 and 6 present the solutions stated by (81) and (89) which are combined dark-bright and periodic singular solitons respectively. 
(e)

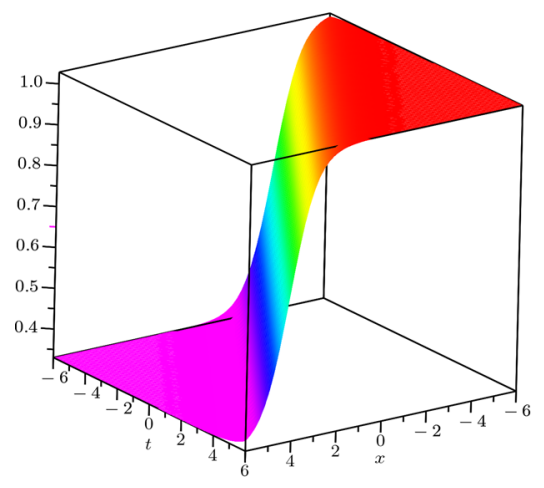

(e-1)

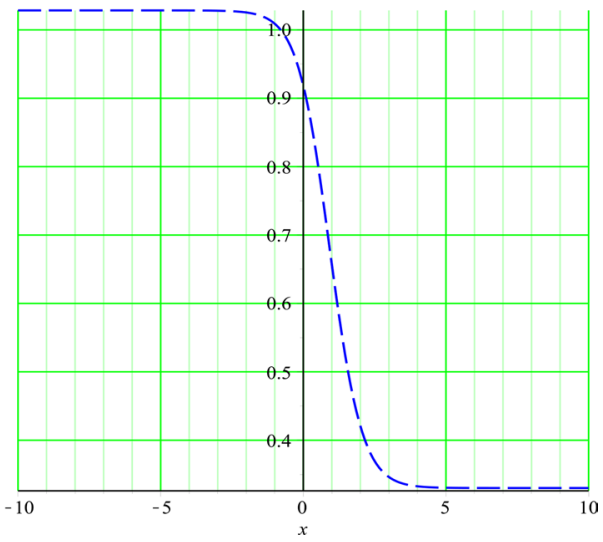

Fig. 5 e 3-d graph of (81) with $h=-0.75, \quad \Lambda_{3}=1.5, \quad \Lambda_{4}=0.8, \quad v=0.65, k=0.9, \theta_{1}=0.57, \quad w=2$ ,$F_{1}=0.5$. (e-1) 2 -d plot of (81) with $t=1$

(f)

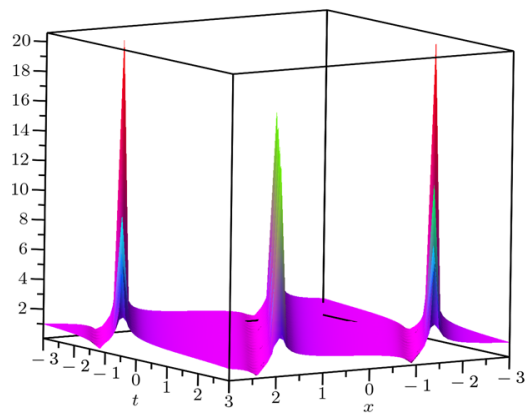

(f-1)

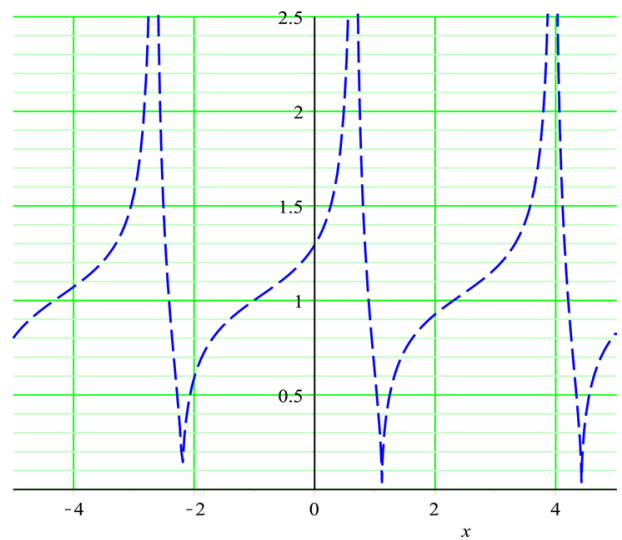

Fig. 6 f 3 -d graph of (89) with $h=0.76, \Lambda_{3}=1.3, \Lambda_{4}=0.9, v=0.66, \quad k=0.7, \theta_{1}=0.59, \quad w=2$, $F_{1}=0.7$. (f-1) 2 -d plot of (89) with $t=1$

\section{Conclusion}

This article includes a comprehensive variety of optical soliton solutions that are discovered for magneto-optic waveguides with AC form of nonlinear refractive index. The outcomes are in the form of bright, singular, dark and combo solitons solutions. These solutions have wide applications in the arena of optoelectronics. As stated in the introduction section, the bright soliton solutions will be a vast advantage in controlling the soliton disorder. This shows that the solitons can be transformed to a shape of parting from a shape of magnetism which would mean clearing the clutter. Consequently, this would carry a factor of "easiness" to the internet bottleneck that is an increasing problem to the present day communications industry where the internet is an important tool for existence. For the 
period of the present pandemic time of COVID-19, where all trade happenings are supervised online, it is bossy to have a flat and continuous flow of pulses for continuous Internet communications. Also, dark solitons are also beneficial for soliton communication when a background wave is exict. Although, singular soliton solutions are only elaborate the shape of solitons and show a total spectrum of soliton solutions created from the model. Furthermore, these novel solutions have many applications in physics and other branches of applied sciences. Further, these solutions may be suitable for understanding the procedure of the nonlinear physical phenomena in wave propagation. We will report these results in future research studies.

\section{References}

Akinyemi, L.: q-Homotopy analysis method for solving the seventh-order time-fractional Lax's Kortewegde Vries and Sawada-Kotera equations. Comp. Appl. Math. 38(4), 1-22 (2019)

Akinyemi, L.: Two improved techniques for the perturbed nonlinear Biswas-Milovic equation and its optical solitons. Optik 243, 167477 (2021)

Akinyemi, L., Iyiola, O.S., Akpan, U.: Iterative methods for solving fourth- and sixth order time-fractional Cahn-Hillard equation. Math. Meth. Appl. Sci. 43(7), 4050-4074 (2020)

Ali Akbar, M., Akinyemi, L., Yao, S.-W., Jhangeer, A., Rezazadeh, H., Khater, M..M..A.., Ahmad, H., Inc, M.: Soliton solutions to the Boussinesq equation through sine-Gordon method and Kudryashov method. Results Phys. 25, 104228 (2021)

Bilal, M., Hu, W., Ren, J.: Different wave structures to the Chen-Lee-Liu equation of monomode fibers and its modulation instability analysis. Eur. Phys. J. Plus 136(4), 1-15 (2021d). https://doi.org/10.1140/ epjp/s13360-021-01383-2

Bilal, M., Ren, J., Younas, U.: Stability analysis and optical soliton solutions to the nonlinear Schrdinger model with efficient computational techniques. Opt. Quantum Electron. 53, 406 (2021c)

Bilal, M., Younas, U., Ren, J.: Dynamics of exact soliton solutions to the coupled nonlinear system with reliable analytical mathematical approaches. Commun. Theor. Phys. 73(8), 085005 (2021a)

Bilal, M., Younas, U., Ren, J.: Dynamics of exact soliton solutions in the double-chain model of deoxyribonucleic acid. Math. Methods Appl. Sci. (2021b). https://doi.org/10.1002/mma.7631

Dai, C.Q., Fan, Y., Wang, Y.Y.: Three-dimensional optical solitons formed by the balance between differentorder nonlinearities and high-order dispersion/ diffraction in parity-time symmetric potentials. Nonlinear Dyn. 98, 489-499 (2019)

Dai, C.Q., Wang, Y.Y., Fan, Y., Zhang, J.F.: Interactions between exotic multi-valued solitons of the $(2+1)$-dimensional Korteweg-de Vries equation describing shallow water wave. Appl. Math. Modell. 80, 506-515 (2020)

Dai, C.Q., Zhou, G.-Q., Chen, R.-P., Lai, X.-J., Zheng, J.: Vector multipole and vortex solitons in twodimensional Kerr media. Nonlinear Dyn. 88, 2629-2635 (2017)

Dotsch, H., Bahlmann, N., Zhuromskyy, O., Hammer, M., Wilkens, L., Gerhardt, R., Hertel, P., Popkov, A.F.: Applications of magneto-optical waveguides in integrated optics: review. J. Opt. Soc. Am. B 22(1), 240-253 (2005)

Fedele, R., Schamel, H., Karpman, V.I., Shukla, P.K.: Envelope solitons of nonlinear Schrodinger equation with an anti-cubic nonlinearity. J. Phys. A 36, 1169-1173 (2003)

Guzman, J.V., Alshaery, A.A., Hilal, E.M., Bhrawy, A.H., Mahmood, M.F., Moraru, L., Biswas, A.: Optical soliton perturbation in magneto-optic waveguides with spatiotemporal dispersion. J. Optoelectron. Adv. Mater. 16(9-10), 1063-1070 (2014)

Haider, T.: A review of magneto-optic effects and its application. Int. J. Electromagn. Appl. 7(1), 17-24 (2017)

Hasegawa, K., Miyazaki, Y.: Magneto-optic devices using interaction between magnetostatic surface wave and optical guided wave. Japenese J. Appl. Phys. 31, 230 (1992)

Imran, M.A., Rehman, H.U., Ullah, N., Akgul, A.: Exact solutions of (2+1)-dimensional Schrodinger's hyperbolic equation using different techniques. Numer. Methods Partial Differ. Equ. (2020). https://doi. org/10.1002/num.22644

Khan, S.: Stochastic perturbation of optical solitons having generalized anti-cubic nonlinearity with bandpass filters and multi-photon absorption. Optik 200, 163405 (2020) 
Khater, M.M., Inc, M., Attia, R.A., Lu, D., Almohsen, B.: Abundant new computational wave solutions of the GM-DP-CH equation via two modified recent computational schemes. J. Taibah Univ. Sci. 14(1), 1554-1562 (2020)

Kudryashov, N.A.: First integrals and general solution of the traveling wave reduction for Schrodinger equation with anti-cubic nonlinearity. Optik 185, 665-671 (2019)

Nestor, S., Houwe, A., Betchewe, G., Inc, M., Doka, S.Y.: A series of abundant new optical solitons to the conformable space-time fractional perturbed nonlinear Schrödinger equation. Phys. Scripta 95, 085108 (2020)

Qiu, Y., Malomed, B.A., Mihalache, D., Zhu, X., Peng, J., He, Y.: Generation of stable multi-vortex clusters in a dissipative medium with anti-cubic nonlinearity. Phys. Lett. A 383(228), 2579-2583 (2019)

Raza, N., et al.: Dynamical analysis and phase portraits of two-mode waves in different media. Results Phys. 19, 103650 (2020)

Raza, N., Arshed, S.: Chiral bright and dark soliton solutions of Schrödinger's equation in $(1+2)$-dimensions. Ain Shams Eng. J. 11, 1237-1241 (2020)

Rehman, H.U., Ullah, N., Imram, M.A.: Optical solitons of Biswas-Arshed equation in birefringent fibers using extended direct algebraic method. Optik 226(2), 15378 (2021b)

Rehman, H.U., Ullah, N., Imran, M.A.: Highly dispersive optical solitons using Kudryashov's method. Optik 199, 163349 (2019)

Rehman, H.U., Ullah, N., Imran, M.A., Akgul, A.: On solutions of the Newell-Whitehead-Segel Equation and Zeldovich Equation. Math. Methods Appl. Sci. 44, 7134-7149 (2021a)

Rehman, H.U., Imram, M.A., Ullah, N., Akgul, A.: Exact solutions of convective-diffusive Cahn-Hilliard equation using extended direct algebraic method. Numerical Methods for Partial Differential Equations, 10 Number (2021c). https://doi.org/10.1002/num.22622

Shang, Y.: The extended hyperbolic function method and exact solutions of the long-short wave resonance equations. Chaos Solitons Fractals 36(3), 762-771 (2008)

Shang, Y., Huang, Y., Yuan, W.: The extended hyperbolic functions method and new exact solutions to the Zakharov equations. Appl. Math. Comput. 200, 110-122 (2008)

Shoji, Y., Mizumoto, T.: Waveguide magneto-optical devices for photonics integrated circuits. Opt. Mater. Express 8(8), 2387-2394 (2018)

Ullah, N., Rehman, H.U., Imran, M.A., Abdeljawad, T.: Highly dispersive optical solitons with cubic law and cubic-quintic-septic law nonlinearities. Results Phys. 17, 103021 (2020)

Wang, Y.Y., Dai, C.Q., Xu, Y.Q., Zheng, J., Fan, Y.: Dynamics of nonlocal and localized spatiotemporal solitons for a partially nonlocal nonlinear Schrodinger equation. Nonlinear Dyn. 92, 1261-1269 (2018)

Wazwaz, A.M.: A study on linear and nonlinear Schrodinger equations by the variational iteration method. Chaos Solitons Fractals 37(4), 1136-1142 (2008)

Yan, Z., Chow, K.W., Malomed, B.A.: Exact stationary wave patterns in three coupled nonlinear Schrodinger/Gross-Pitaevskii equations. Chaos Solitons and Fractals 42(5), 3013-3019 (2009)

Younas, U., Ren, J.: Investigation of exact soliton solutions in magneto-optic waveguides and its stability analysis. Results Phys. 21, 103816 (2021). https://doi.org/10.1016/j.rinp.2021.103816

Zayed, E.M.E., Shohib, R.M.A., El-Horbaty, M.M., Biswas, A., Asma, M., Ekici, M., Alzahrani, A.K., Belic, M.R.: Solitons in magneto-optic waveguides with quadratic cubic nonlinearity. Phys. Lett. A 384(25), 126456 (2020)

Zayed, E.M.E., Alngar, M.E.M., El-Horbaty, M.M., Biswas, A., Guggilla, P., Ekici, M., Alzahrani, A.K., Belic, M.R.: Solitons in magneto-optic waveguides with parabolic law nonlinearity. Optik 222, 165314 (2020)

Publisher's Note Springer Nature remains neutral with regard to jurisdictional claims in published maps and institutional affiliations. 